\title{
Adherence to Endotracheal Tube Depth Guidelines and Incidence of Malposition in Infants and Children
}

\author{
Teresa A Volsko MBA MHHS RRT CMTE FAARC, Neil L McNinch MSc RN, \\ Donald S Prough MD, and Michael T Bigham MD
}

\begin{abstract}
BACKGROUND: Adherence to guidelines for endotracheal tube (ETT) insertion depth may not be sufficient to prevent malposition or harm to the patient. To obtain an estimate of ETT malpositioning, we evaluated initial postintubation chest radiographs and hypothesized that many ETTs in multiple intubation settings would be malpositioned despite adherence to Pediatric Advanced Life Support and Neonatal Resuscitation Program guidelines. METHODS: In a random subset (randomization table) of 2,000 initial chest radiographs obtained from January 1, 2009, to May 5, 2012, we recorded height, weight, age, sex, ETT inner diameter, and $\mathrm{cm}$ marking at the lip from the electronic health record. Chest radiographs of poor quality and with spinal or skeletal deformities were excluded. We defined adherence to Pediatric Advanced Life Support or Neonatal Resuscitation Program guidelines as the difference between predicted and actual ETT markings at the lip as \pm 0.25 , \pm 0.50 , or $\pm 1.0 \mathrm{~cm}$ for ETTs of $2.5-4,4.5-6.0$, or $>6.5 \mathrm{~mm}$ inner diameter, respectively. We defined the proper position as the ETT tip being below the thoracic inlet (superior border of the clavicular heads) and $\geq 1 \mathrm{~cm}$ above the carina. Descriptive statistics reported demographics, guideline adherence, and malposition incidence. The chi-square test was used to assess relationships among intubation setting, malposition, and depth guideline adherence $(P<.05$, significant). RESULTS: We reviewed 507 records, 477 of which met inclusion criteria and had sufficient data for analysis. Fifty-six percent of the subjects were male, with median (interquartile range) age $15.2(3.4-59.4)$ months, and 330 ETTs $(69 \%)$ were malpositioned: 39 above the thoracic inlet, and $291<1 \mathrm{~cm}$ above the carina. Of 79 ETTS (17\%) that adhered to depth guidelines, 56 (74\%) were malpositioned. Three-hundred seventy-three ETTs $(83 \%)$ did not meet guidelines. Two-hundred sixty-four $(68 \%)$ were malpositioned. The intubation setting did not influence malposition or guideline adherence $(P=.54)$. CONCLUSIONS: In infants and children, a high proportion of ETTs were malpositioned on the first postintubation chest radiograph, with little influence of guideline adherence. Key words: intubation; tracheal tube malposition; PALS; NRP; pediatrics. [Respir Care 2018;63(9):1111-1117. (C) 2018 Daedalus Enterprises]
\end{abstract}

\section{Introduction}

Airway management is essential to the care of infants and children who are critically ill. To protect the airway

\footnotetext{
Ms Volsko is affiliated with the Nursing Administration, Akron Children's Hospital Akron, Ohio, and the Rebecca D Considine Research Center, Akron Children's Hospital, Akron, Ohio. Mr McNinch is affiliated with Akron Children's Hospital, Akron, Ohio. Dr Prough is affiliated with the Department of Anesthesiology, University of Texas Medical Branch at Galveston, Galveston, Texas. Dr Bigham is affiliated with the Division of Critical Care Medicine, Akron Children's Hospital, Akron, Ohio, and the College of Medicine, Northeast Ohio Medical University, Rootstown, Ohio.
}

and provide invasive ventilatory support, an endotracheal tube (ETT) must be properly placed in the mid-tracheal position. ${ }^{1}$ However, there are challenges to initially plac-

\footnotetext{
Ms Volsko presented this research at Society of Critical Care Medicine (SCCM) 2016.

Dr Bigham, Dr Prough, and Ms Volsko disclose a relationship with Prospiria. The other authors have no conflicts to disclose.

Correspondence: Teresa A Volsko MBA MHHS RRT CMTE FAARC, Nursing Administration, Akron Children's Hospital, Akron, Ohio 44308. E-mail: tvolsko@chmca.org
}

DOI: $10.4187 /$ respcare. 06024 
ing and maintaining an ETT in the correct position. The tracheal length ranges from 4 to $6 \mathrm{~cm}$ in infants and small children and from 5 to $10 \mathrm{~cm}$ in larger children. ${ }^{2}$ The relatively short tracheal length of infants and small children complicates proper initial positioning of the tip of an ETT and subsequent maintenance of the proper position. Malposition may be exacerbated by changes in the head position, such as neck flexion or extension, which causes the ETT to migrate cauda with neck flexion and cephalad with neck extension. ${ }^{3}$

Significant morbidity and mortality are associated with malpositioned ETTs. Shallowly placed tubes can contribute to unplanned extubation as well as an improper position of an inflated cuff between the vocal cords. ${ }^{3}$ Irritation of the carina and endobronchial intubation may occur when an ETT is placed too deeply. Malpositioned ETTs that lead to unplanned extubations often result in emergent, endotracheal re-intubation, which can be life threatening if the airway cannot be secured promptly. ${ }^{4}$ Repeated intubations, especially those performed emergently, increase the risk of laryngeal or tracheal injury and scarring. Endobronchial intubations impair ventilation by excessively ventilating one lung and inadequately ventilating the other. ${ }^{5}$

To minimize the risk of placing an ETT in a suboptimal position, depth marks are located at the ETT tip as a visual guide for the clinician performing the tracheal intubation. During intubation, placing an ETT with the depth mark just below the vocal cords will theoretically position it in the mid-trachea. ${ }^{6}$ To further reduce risk, the American Academy of Pediatrics through the Neonatal Resuscitation Program $^{7}$ provides guidelines for ETT insertion depth for infants up to $28 \mathrm{~d}$ of age. Depth guidelines were available for infants and children in the 2011 edition of Pediatric Advanced Life Support ${ }^{8}$ (PALS), which is still the standard practice at our institution. To obtain an estimate of the incidence of ETT malposition in our tertiary care pediatric hospital, we evaluated initial postintubation portable chest radiographs of infants and children admitted to our pediatric ICU. We hypothesized that many ETTs would be malpositioned, regardless of adherence to the guidelines specified in the 2011 edition of PALS ${ }^{8}$ and depth guidelines recommended by the Neonatal Resuscitation Program. ${ }^{7}$

\section{Methods}

\section{Setting and Study Design}

This retrospective single-institution study was performed in the pediatric ICU at Akron Children's Hospital, Akron, Ohio. The protocol was approved by the institutional review board, with a waiver of informed consent because it was a retrospective records review with no intervention or interaction with the subjects. Infants and children (new-

\section{QUICK LOOK}

\section{Current knowledge}

The relatively short tracheal length of infants and small children makes it difficult to place the endotracheal tube (ETT) in the proper position following endotracheal intubation. Changes in head position, such as neck flexion or extension, cause the ETT to migrate caudal with neck flexion and cephalad with neck extension, which further hinders the ability to maintain a proper position of the ETT within the trachea. A malpositioned ETT can cause significant morbidity and mortality.

\section{What this paper contributes to our knowledge}

Although initially placing and maintaining proper ETT placement is an essential component of safe and effective care, many ETTs are malpositioned. The use of Neonatal Resuscitation Program and Pediatric Advanced Life Support (PALS) guidelines did not improve the likelihood of correctly placed ETTs. We found a significant relationship between gender and correct positioning with PALS and/or Neonatal Resuscitation Program guideline adherence, which is an unexpected finding and an association that has not yet been reported in the literature.

born to $16 \mathrm{y}$ of age) who required tracheal intubation and mechanical ventilatory support met inclusion criteria only if the electronic health record contained an order for a post-intubation chest radiograph to confirm the ETT position. Exclusion criteria were the following: intubated and mechanically ventilated children for whom a chest radiograph was not ordered, those children with spinal deformities (eg, lordosis, kyphosis, scoliosis, or kyphoscoliosis), suspected skeletal dysplasia (achondroplasia), and radiographs of poor quality (tip of the ETT or the carina could not be easily identified).

A random table was used to select a subset of the 2,000 infants and children admitted to the pediatric ICU from January 1, 2009, to May 5, 2012 (Fig. 1). A power analysis was performed a priori to determine the number of subjects required for a chi-square test, with $P<.05$ as statistically significant, and power $=80 \%$. The electronic health record was used to abstract demographic data, including height, weight, body surface area, age, and sex. The ETT size, inner diameter, and insertion depth (cm marking at the lip) were also recorded from the electronic health record. The estimated depth of insertion was calculated for each subject by using the PALS ${ }^{8}$ guidelines for those $\geq 28 \mathrm{~d}$ of age and the Neonatal Resuscitation Program ${ }^{7}$ guidelines for those $\leq 28 \mathrm{~d}$ of age (Table 1). 


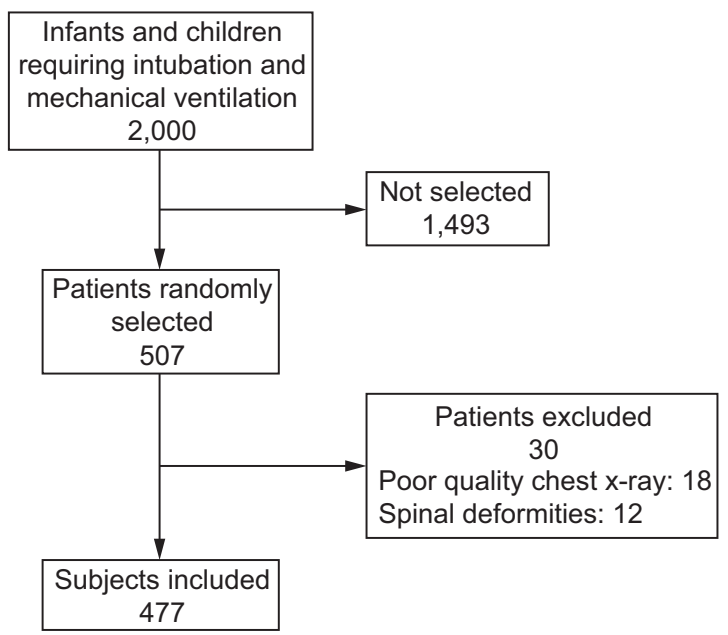

Fig. 1. Consort diagram of study subjects.

Table 1. Guidelines Used to Determine Depth of Endotracheal Tube Insertion

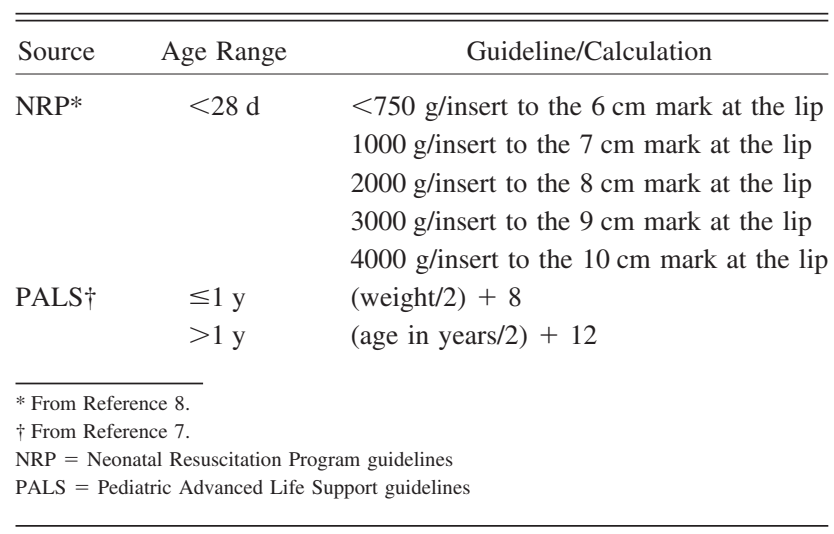

Documentation of the ETT $\mathrm{cm}$ marking at the lip was collected and compared with depth recommendations calculated by using the 2011 PALS guidelines ${ }^{8}$ or current Neonatal Resuscitation Program ${ }^{7}$ guidelines. Adherence to the aforementioned guidelines was defined as the difference between predicted and actual ETT markings at the lip within a clinically acceptable tolerance level or level of error that was determined by consensus of a group of pediatric intensivists and pediatric radiologists who evaluate imaging and report chest radiographic results at our institution (Table 2). The first anteroposterior supine chest radiograph obtained after intubation that satisfied inclusion criteria was used to determine the position of the ETT. As a standard, children had been positioned supine with the head held in a midline position. The ETT marking at the lip was documented in the electronic health record at the time of the chest radiograph.

If a chest radiograph was not available immediately after intubation (ie, for intubations that occurred in the operating room or at a referral institution), the principle
Table 2. Tolerance Levels Used to Determine Staff Adherence to Depth Insertion Guidelines

\begin{tabular}{lc}
\hline \hline $\begin{array}{c}\text { Endotracheal Tube, } \\
\text { Inner Diameter }(\mathrm{mm})\end{array}$ & Tolerance Level $(\mathrm{mm})$ \\
\hline $2.5-4.0$ & \pm 2.5 \\
$4.5-6.0$ & \pm 5.0 \\
$\geq 6.5$ & \pm 10.0 \\
\hline
\end{tabular}

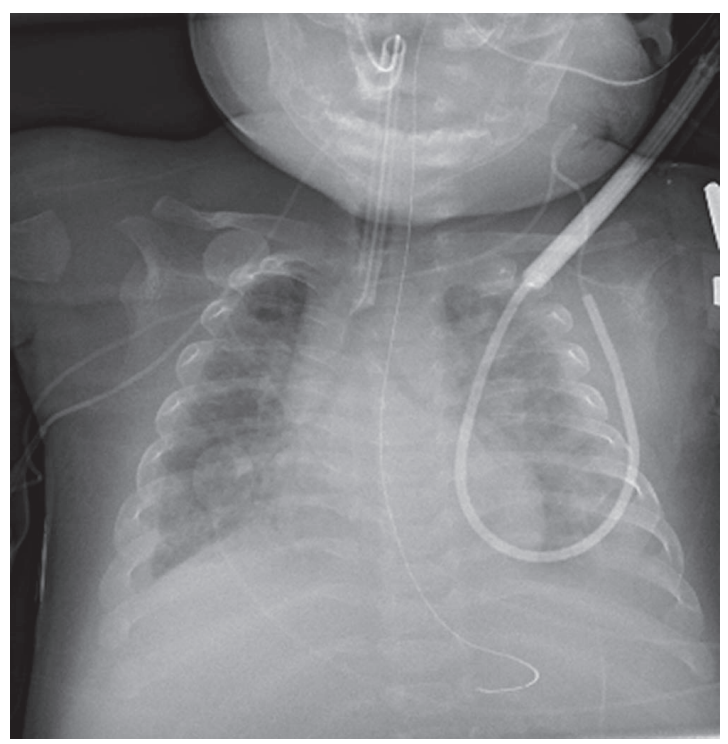

Fig. 2. Chest radiograph, illustrating an endotracheal tube identified to be in the correct position by radiographic evaluation.

investigator verified that the ETT marking at the lip documented in the electronic health record at the time of intubation was identical to the ETT marking at the lip documented at the time the chest radiograph was obtained on arrival and admission to our pediatric ICU. Results of the radiologist's interpretation of the ETT position were used to identify the position of the ETT within the trachea and determine if criteria for the proper position were met. We defined the proper or mid-tracheal position as the ETT tip being below the thoracic inlet, identified as the superior border of the clavicular heads, and $\geq 1 \mathrm{~cm}$ above the carina (Fig. 2). In cases in which the ETT position was described qualitatively, the radiographic image was retrieved and reviewed by a radiologist to quantitatively determine and record the position of the ETT.

\section{Analytical Methods}

Data were collected and entered into Excel (Microsoft, Redmond, California), de-identified, and exported to SAS (SAS Institute, Cary, North Carolina) for statistical analyses. Descriptive statistics were used to report demographic and clinical data, including Neonatal Resuscitation Pro- 
Table 3. Summary of Demographic, Clinical, and Anthropometric Characteristics

\begin{tabular}{lccccc}
\hline \hline \multicolumn{1}{c}{ Variable } & $n$ & Median & IQR & Minimum & Maximum \\
\hline Age, mo & 477 & 15.2 & $3.4-59.4$ & 0 & 345.6 \\
BSA, m ${ }^{2}$ & 391 & 0.4 & $0.3-0.7$ & 0.1 & 1.9 \\
Weight, kg & 477 & 10 & $5.3-18.1$ & 1.9 & 80.0 \\
Height, cm & 392 & 72 & $56-99.5$ & 42 & 185 \\
PICU LOS, d & 477 & 4.5 & $2.1-9.8$ & 0.1 & 190.8 \\
Time intubated, d & 477 & 1.91 & $0.61-4.85$ & 0.01 & 41.7 \\
Cuffed ETT size, mm & 471 & 4 & $3.5-5$ & 3 & 8 \\
& & & & & \\
IQR $=$ interquartile range & & & & & \\
BSA = body surface area & & & & & \\
PICU = pediatric ICU & & & & & \\
LOS = length of stay & & & & & \\
ETT $=$ endotracheal tube & & & & & \\
&
\end{tabular}

$\operatorname{gram}^{7}$ and PALS ${ }^{8}$ guideline adherence, and the incidence of ETT malposition. The chi-square test of independence assessed potential relationships for sex, intubation setting, and guideline adherence with malposition. Receiver operating characteristic curve analysis was performed to assess the diagnostic value of the lip calculations (based on PALS/Neonatal Resuscitation Program guidelines) for malposition. Statistical significance was established a priori at a type-1 error rate of alpha $=0.05$.

\section{Results}

We reviewed the radiologist's interpretation of chest radiographs and electronic health records from 507 randomly selected intubated infants and children, of whom 477 met inclusion criteria and had sufficient data for analysis: $267(56 \%)$ were male and $210(44 \%)$ were female. The median (interquartile range [IQR]) age was 15.2 (3.4$59.4)$ months, median weight was $10.0(5.3-18.1) \mathrm{kg}$, median height was $72.0(56.0-99.5) \mathrm{cm}$, and median body surface area was $0.4(0.3-0.7) \mathrm{m}^{2}$. Five percent $(n=24)$ of the radiologist's reports only provided a qualitative description of the ETT position and required a radiologist to review the chest radiograph and provide distance measure of the ETT tip from the carina or above the thoracic inlet.

All the subjects were intubated with a cuffed ETT. ETT sizes ranged from 3.0 to $8.0 \mathrm{~mm}$ inner diameter, with a median (IQR) of $4.0(3.5-5.0) \mathrm{mm}$ inner diameter. The median (IQR) for device days was $1.9(0.6-4.9)$, and the median (IQR) for pediatric ICU stay was 4.5 (2.1-9.8) d. A summary of the demographic, clinical, and anthropometric characteristics is found in Table 3. The first chest radiograph after intubation identified improperly placed ETTs (malposition) in 330 of 477 subjects (69\%), whereas only 147 subjects $(31 \%)$ had ETTs positioned properly (Fig. 3). Twelve percent of malpositioned ETTs $(n=39)$ were too shallowly inserted or the ETT tip was above the thoracic inlet. Eighty-eight percent $(n=291)$ of malpositioned ETTs were too deeply inserted or the ETT tip was $<1.0 \mathrm{~cm}$ from the carina or was endobronchial (Fig. 3).

The correct position, defined as adherence to the aforementioned depth guidelines, within tolerance levels (identified in Table 2), could be determined for 467 of the 477 subjects. Documentation of the $\mathrm{cm}$ marking at the lip was missing in 10 cases, in children intubated in the pediatric ICU, who were subsequently excluded from this portion of the analysis. Of the ETTs that adhered to depth criteria (79/467 [17\%]), those in which the mark at the lip was correct, 58 of 79 (73\%) were shown to be malpositioned by chest radiograph. Of nonadherent ETTs or those in which the mark at the lip was incorrect, 264 of 388 (68\%) were malpositioned.

The site of the initial intubation was documented in 458 subjects. A plurality of intubations occurred at outside or referral facilities $(n=211$ [46\%]). Of the intubations that occurred at referral facilities, a majority of the intubations $(n=208$ [99\%]) were performed by a registered respiratory therapist, who was a member of our neonatal/pediatric specialty transport team. The remaining intubations occurred at our institution in the pediatric ICU $(n=136$ [30\%]) or the operating rooms $(n=113$ [25\%]). There was no record of a chest radiograph immediately after intubation at the referral facility or in the operating room at our facility. In these cases, the results reported were reflective of the adherence to verifying the ETT marking at the lip, documented in the electronic health record at the time of intubation matched the marking at the lip when the chest radiograph was obtained on admission to the pediatric ICU.

Results of the chi-square test of independence indicated that there was not a statistically significant relationship between malposition (by chest radiograph) and adherence to depth guidelines for correct ETT placement (chisquare $=0.9, P=.34$ ). There was no evidence of a significant relationship between malposition and sex (chisquare $=0.4, P=.53)$, malposition and the site of initial intubation (chi-square $=0.9, P=.61$ ), or correct position based on depth guidelines and site of initial intubation (chi-square $=1.2, P=.54$ ).

There, however, was evidence of a significant relationship between the correct position and sex (chisquare $=5.4, P=.02$ ), with an associated odds ratio and 95\% CI 1.8 (1.1-2.9); analysis of these data indicates that male subjects may have been more likely to experience incorrect positioning than the female subjects when depth guidelines are used. Receiver operating characteristic curve analysis results indicated markings at the lip, which are specified by PALS $^{8}$ and Neonatal Resuscitation Program ${ }^{7}$ guidelines and were used to determine the correct placement, were a poor indicator of 


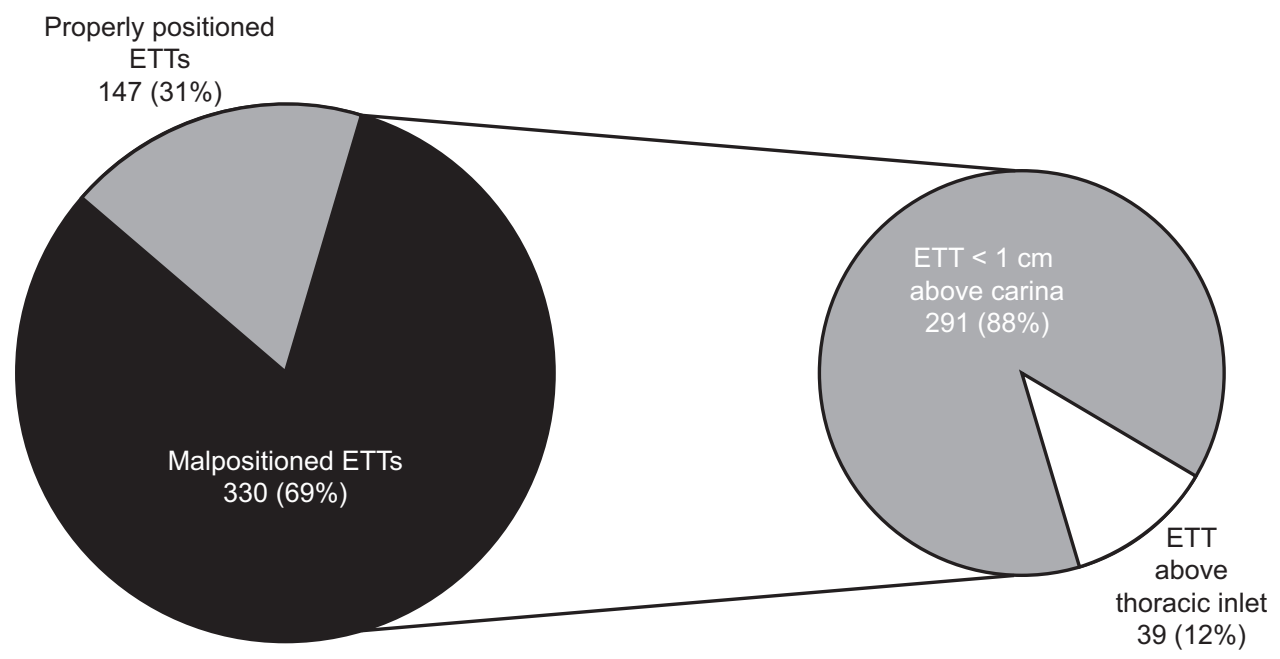

Fig. 3. Occurrence of malpositioned endotracheal tubes (ETT) after intubation in infants and children who require mechanical ventilatory support in the pediatric ICU.

proper ETT position (area under the curve $=0.65,95 \%$ CI 0.59-0.70).

\section{Discussion}

Intubation is a procedure that requires the skills to place an ETT within the trachea and at the proper depth. It is also important for bedside providers, such as respiratory therapists, nurses, physicians, and advanced practice providers, to minimize tube migration during care. Achieving and maintaining proper ETT insertion depth are challenging but critical for safe and effective care. Significant morbidity and mortality are associated with improperly positioned ETTs, including atelectasis, hypoxemia, pneumothorax, hypotension, and cardiac arrhythmias. ${ }^{9}$

ETT depth guidelines were designed to minimize the risk of placing an ETT in an improper position within the trachea. These guidelines use easily memorable, simple calculations based on anthropometric measures to determine the depth of ETT insertion by placing calculated depth marking at the lip. Although our practice is to follow Neonatal Resuscitation Program ${ }^{7}$ and the PALS ${ }^{8}$ guidelines published in 2011 for depth of ETT position during intubation and with airway care, those guidelines were followed in a minority of initial intubations (17\%) at our institution and by providers at referring community hospitals or academic medical centers. The current PALS curriculum no longer includes the calculations used to estimate the depth of ETT insertion. ${ }^{10}$ However, our results indicated that the use of Neonatal Resuscitation Program and PALS guidelines did not improve the likelihood of correctly placed ETTs. Our findings did not support our current practice of continuing to use the 2011 PALS $^{8}$ depth guidelines. Lau et al ${ }^{11}$ reported that the 2011 PALS depth insertion guidelines underestimated appropriate tracheal tube lengths in children $>1 \mathrm{y}$ of age, which could account for a greater propensity for malposition when the aforementioned guidelines were followed. We found no statistically significant relationship between adherence to depth guidelines and the incidence of ETT malposition. Receiver operating characteristic curve analysis confirmed that use of the aforementioned guidelines did not improve the incidence of placing the ETT tip in the appropriate intratracheal position.

In a prospective study of 225 children $<12$ y of age, Phipps et $\mathrm{al}^{12}$ found that $23 \%$ of ETTs were malpositioned, most of which were placed too deeply, when PALS ${ }^{8}$ guidelines were used to calculate insertion depth. Although our incidence of malposition was much higher, Phipps et al ${ }^{12}$ considered ETTs acceptably placed if the tip was $0.5-1.0 \mathrm{~cm}$ from the carina, whereas we defined properly placed ETTs as $>1.0 \mathrm{~cm}$ from the carina. Our more conservative definition accounted for the relatively short length of the trachea in infants and small children (4$6 \mathrm{~cm})^{1}$ and in larger children $(5-10 \mathrm{~cm}) .^{2}$ It also accounted for the manner in which the infant and/or child was positioned for the portable chest radiograph. Our subjects were positioned with the head held at the midline when positioned on the radiographic plate. However, during routine care, an infant or child in the pediatric ICU may have the head repositioned during care, which can change the intratracheal position of the ETT. When the tip of the ETT is closer to the carina (ie, $<1 \mathrm{~cm}$ ), repositioning the child's head may result in flexion that causes the ETT tip to migrate to the carina or main bronchus. Similar to observations by Phipps et al, ${ }^{12}$ a majority of malpositioned tubes in our study were placed too deeply. 
Peterson et $\mathrm{al}^{13}$ reported inaccuracies with the use of Neonatal Resuscitation Program ${ }^{7}$ depth guidelines, or the 7-8-9 rule, for very low birthweight infants. ETTs were placed too deeply by $0.3-0.93 \mathrm{~cm}$ in infants who weighed $<750 \mathrm{~g}$ when Neonatal Resuscitation Program guidelines were used. Under- and overestimated depths of insertion can potentially result in clinically important consequences. Airway compromise due to unplanned extubation and mainstem intubation accounted for $23 \%$ of adverse respiratory events among the pediatric population, including death $(41 \%)$ and brain damage (21\%). ${ }^{14}$ Koshy et a ${ }^{15}$ found the mid-tracheal position to be the optimal and, when positioning was directed by the use of chest radiography, resulted in a majority $(98.5 \%)$ of correctly positioned ETTs after intubation in the operating room.

The significant relationship between sex and correct positioning with PALS and/or Neonatal Resuscitation Program guidelines adherence is an unexpected finding and an association that has not yet been reported in the literature. Additional research is needed to determine if estimations of tracheal length differ with sex, similar to differences sex plays when estimating or calculating ideal body weight. Evaluating outcome variables retrospectively provided a realistic view of our practice for verifying the initial placement of the ETT within our institution and at our referral facilities. This information is helpful in identifying process gaps from which quality improvement initiatives can be derived. The criteria for malposition was well defined, and uniformly applied, and required a quantitative description of the ETT position by a radiologist, who facilitates identification of malpositioned ETTs.

\section{Limitations}

This study was a single-institution retrospective analysis. One limitation included poor adherence to depth insertion guidelines, which may have introduced bias. Although this study was well powered for the primary analysis (evaluation of the relationship between malposition and adherence to depth guidelines) by the a priori conduct of sample-size estimation, it may not have been fully powered for the secondary exploratory analyses conducted (on anthropomorphic and clinical characteristics). Therefore, these results may not be fully generalizable, and further study with a sufficiently powered sample would be needed to validate or refute the findings.

There were 10 instances in which the bedside respiratory therapist did not document the $\mathrm{cm}$ marking at the lip, which precluded determination of whether the Neonatal Resuscitation Program or PALS guidelines were followed. This study did not verify how the clinician who performed the intubation calculated the initial depth of insertion. As a result, we were unable to account for potential mathematical errors (calcula- tion errors, use of the wrong formula), which have the potential to position an ETT at the incorrect marking at the lip.

It is important to note that the brand of ETT was not standardized in our retrospective study. Infants and children who were intubated while at referring community hospitals and academic medical centers were also included in this study; the commercial brand of ETT used in those cases was not documented and may have differed from that used at our institution. The position of cuffs on ETT is not standardized, can vary by ETT brand, and can influence the incidence of malposition. ${ }^{14,16}$

\section{Conclusions}

In infants and children, a high proportion of ETTs are malpositioned after intubation, many of which have the potential to cause harm. Use of Neonatal Resuscitation Program and PALS guidelines did not improve the incidence of malposition. There is the opportunity for further research to refine and test modifications of current insertion depth guidelines.

\section{REFERENCES}

1. Wessels LF, Reinders MJ, Hart AA, Veenman CJ, Dai H, He YD, van't Veer LJ. A protocol for building and evaluating predictors of disease state based on microarray data. Bioinformatics 2005;21(19): 3755-3762.

2. Griscom NT, Wohl ME. Dimensions of the growing trachea related to age and gender. AJR Am J Roentgenol 1986;146(2):233-237.

3. Sugiyama K, Yokoyama K. Displacement of the endotracheal tube caused by change of head position in pediatric anesthesia: evaluation by fiberoptic bronchoscopy. Anesth Analg 1996;82(2):251-253.

4. Veldman A, Trautschold T, Weiss K, Fischer D, Bauer K. Characteristics and outcome of unplanned extubation in ventilated preterm and term newborns on a neonatal intensive care unit. Paediatr Anaesth 2006;16(9):968-973.

5. Mahajan A, Hoftman N, Hsu A, Schroeder R, Wald S. Continuous monitoring of dynamic pulmonary compliance enables detection of endobronchial intubation in infants and children. Anesth Analg 2007; 105(1):51-56.

6. Orive G, Anitua E, Pedraz JL, Emerich DF. Biomaterials for promoting brain protection, repair and regeneration. Nat Rev Neurosci 2009;10(9):682-692.

7. Weiner GM, editor. Textbook of Neonatal Resuscitation, 6th ed. Dallas: American Heart Association and American Academy of Pediatrics. Elk Grove Village, IL:American Academy of Pediatrics; 2011.

8. Chameides L, Samson RA, Schexnayder SM, Hazinski MF, editors. Pediatric Advanced Life Support: Provider Manual. American Heart Association and American Academy of Pediatrics; 2011.

9. Fiadjoe JE, Nishisaki A, Jagannathan N, Hunyady AI, Greenberg RS, Reynolds PI, et al. Airway management complications in children with difficult tracheal intubation from the Pediatric Difficult Intubation (PeDI) registry: a prospective cohort analysis. Lancet Respir Med 2016;4(1):37-48.

10. de Caen AR, Berg MD, Chameides L, Gooden CK, Hickey RW, Scott HF, et al. Part 12: Pediatric Advanced Life Support: 2015 American Heart Association Guidelines Update for Cardiopulmo- 


\section{Adherence to ETT Depth Guidelines in Pediatrics}

nary Resuscitation and Emergency Cardiovascular Care. Circulation 2015;132(18 Suppl 2):S526-S542.

11. Lau N, Playfor SD, Rashid A, Dhanarass M. New formulae for predicting tracheal tube length. Paediatr Anaesth 2006;16(12):1238-1243.

12. Phipps LM, Thomas NJ, Gilmore RK, Raymond JA, Bittner TR, Orr RA, Robertson CL. Prospective assessment of guidelines for determining appropriate depth of endotracheal tube placement in children. Pediatr Crit Care Med 2005;6(5):519-522.

13. Peterson J, Johnson N, Deakins K, Wilson-Costello D, Jelovsek JE, Chatburn R. Accuracy of the 7-8-9 Rule for endotracheal tube placement in the neonate. J Perinatol 2006;26(6):333-336.
14. Jimenez N, Posner KL, Cheney FW, Caplan RA, Lee LA, Domino KB. An update on pediatric anesthesia liability: a closed claims analysis. Anesth Analg 2007;104(1):147-153.

15. Koshy T, Misra S, Chatterjee N, Dharan BS. Accuracy of a chest $\mathrm{X}$-ray-based method for predicting the depth of insertion of endotracheal tubes in pediatric patients undergoing cardiac surgery. J Cardiothorac Vasc Anesth 2016;30(4):947-953.

16. Weiss M, Balmer C, Dullenkopf A, Knirsch W, Gerber ACh, Bauersfeld U, Berger F. Intubation depth markings allow an improved positioning of endotracheal tubes in children. Can J Anaesth 2005;52(7):721-726.

This article is approved for Continuing Respiratory Care Education credit. For information and to obtain your CRCE

(free to AARC members) visit 